\title{
Effects of virtual reality on social participation after stroke: a systematic review
}

\author{
Letícia Costa Queiroz ${ }^{1}$, Kênia Kiefer Parreiras de Menezes ${ }^{1,2}$, Patrick Roberto Avelino ${ }^{1}$
}

\begin{abstract}
Background: The cerebrovascular accident (CVA), or stroke, is characterized as a vascular injury occurring in the nervous tissue, in which there is interruption in the blood flow with consequent loss of the local supply of nutrients and oxygen, leading to tissue death. According to data from the Unified Health System and other studies, the CVA represents the greatest cause of death and permanent disability in Brazil. Objectives: The objective of this study was to perform a systematic review of the literature, searching for evidence of the effects of the use of the rehabilitation based on virtual reality to help recover the social participation of hemiparetic subjects after stroke. Methods: Searches were conducted on Medline, CINAHL, Web of Science, LILACS and PEDro databases, without restriction regarding the language and publication year of the study. The articles were evaluated by title, abstract and full text and, finally, selected in relation to the eligibility by two independent authors. The methodological quality of the included experimental studies was evaluated according to the PEDro scale. Results: Among the 11 articles included in the present review, only 4 demonstrated significant effects of virtual reality training on social participation/quality of life of hemiparetic subjects, when compared to the control group. Conclusion: The studies analyzed show that although there seems to be benefits of using virtual reality in global aspects of hemiparetic patient recovery, such as in motor function, the results are still not consistent regarding the social participation.
\end{abstract}

Keywords: Stroke; Virtual Reality; Social Participation.

\section{INTRODUCTION}

The cerebrovascular accident (CVA), or stroke, is characterized as a vascular injury occurring in the nervous tissue, in which there is interruption in the blood flow with consequent loss of the local supply of nutrients and oxygen, leading to tissue death $^{(1)}$. All age groups may be affected and the consequences of the event in general depend on the area affected and the degree of severity of the injury ${ }^{(2)}$. Usually the sequelae present itself in the hemisphere opposite to the hemisphere reached, preserving most of the functions on the ipsilateral side to the lesion $^{(2)}$. According to data from the Unified Health System and other studies, the CVA represents the greatest cause of death and permanent disability in $\operatorname{Brazi}^{(2,3)}$.

Among the sequelae present in the survivors, there is usually muscle weakness, motor control impairment, sensory deficit, balance deficit, spasticity, asymmetry in the discharge of weight in the lower limbs and proprioceptive deficits ${ }^{(4)}$. Because of that, these subjects present difficulties in ambulation, limitations in the execution of activities of daily living and, consequently, remain restricted to their home, without involvement with the community, with restrictions on social participation ${ }^{(5)}$.

Maintaining social participation is important because it favors patients' motivation and adherence to treatments, preventing psychological dysfunctions such as depression and dementia, and promoting a sense of self-efficacy, which are extremely relevant factors in the quality of life of patients ${ }^{(6,7)}$. Given this, it is necessary to seek interventions that are effective in helping the functional recovery and social participation of these patients, promoting their reintegration into the community. One of the resources recently explored is virtual reality, a tool that has proved to be efficient in the rehabilitation of hemiparetic patients ${ }^{(5)}$.

Virtual reality is defined as an multimedia and immersive environment, in which computers can simulate the physical presence of real or imaginary locations, as well as recreate sensory experiences ${ }^{(4)}$. It is considered an interactive and motivational resource that allows the therapist to modulate and manipulate the intensity and feedback of the proposed activities in an individualized way, according to the needs of the patient ${ }^{(3)}$. Some studies have shown that the use of the adequate platform allows training the skills necessary to the individual in his daily life, making the use of virtual reality promote cortical reorganization and improved coordination and balance. You et al., in one of the first trials on the subject, investigated the effects of the use of the therapy based on virtual reality in the motor recovery of patients with chronic stroke. In a sample of 10 patients, the authors performed qualitative and quantitative gait analyzes, as well as the

Corresponding Author: Kênia Kiefer Parreiras de Menezes. Adress: Av. Pres. Antônio Carlos, 6627 - Pampulha, Belo Horizonte (MG), Brasil. CEP: $31270-901$.

Telephone: (31) 3309-7403 keniakiefer@yahoo.com.br

1 Universidade Federal de Minas Gerais (UFMG), Belo Horizonte (MG), Brasil

2 Fundação Comunitária de Ensino Superior de Itabira (FUNCESI), Itabira (MG), Brasil

Financial source: The authors declare that there was no financial source.

Submission date 05 January 2018; Acceptance date 03 July 2018; Publication date 15 October 2018 
verification of magnetic resonance imaging before and after the intervention. The authors demonstrated that after four weeks of intervention the gait-related tests showed significant changes in score, and magnetic resonance imaging indicated suggestive signs of cortical reorganization in the supplemental motor area in the experimental group ${ }^{(5)}$.

Although the results of randomized clinical trials appear promising, the systematic review is the gold standard study to answer the scientific questions, because it summarizes all available literature on the subject using clear criteria of inclusion and exclusion of works to avoid biases and conflicts of interests, elements not necessarily present in other types of studies ${ }^{(8)}$. However, no reviews were found investigating the effects of virtual reality on the social participation of subjects after stroke. Therefore, the objective of this study was to carry out a systematic review of the literature, searching for evidence of the effects of the use of rehabilitation based on virtual reality in the recovery of the social participation of hemiparetic individuals after stroke.

\section{METHODS}

\section{Design}

This study is a systematic review of the literature, with the objective of describe the evidence on the effects of the use of rehabilitation based on virtual reality in the recovery of social participation of hemiparetic individuals after stroke.

\section{Procedures}

An online search was performed in the databases Medline, CINAHL, Web of Science, LILACS and PEDro, using the combination of words "Virtual Reality, Rehabilitation, Stroke, Cva, Hemiplegia, Hemiparesis, Hemiparetic, Paresis, Plegia, Paretic, Ischemic and Hemorrhagic", and their respective terms in Portuguese. Two authors performed the research independently, initially by analyzing the titles and abstracts of the found articles. Subsequently, among the papers selected, the complete texts were read. The final choice of included articles was completed after a third independent evaluator resolved the disagreements between the first two reviewers.

\section{Inclusion Criteria}

The included articles had therapy based on virtual reality as intervention in hemiparetic patients, after acute, subacute or chronic stroke, focusing on the outcomes related to social participation, without restrictions of age or gender of participants. Studies that evaluated the quality of life were also included as a possible outcome measure for social participation. There was no restriction on the publication date or language.

\section{Data Collection}

The data were extracted and arranged in a standard table, adapted from the Cochrane Collaboration. The information extracted from the studies was: study type, characteristics of the included participants (as gender, age, sample size, among others), objective, intervention protocol (as duration, intensity, frequency, description of activities, among others), the used outcome measures and the found results.

\section{Methodological quality}

The methodological quality of the clinical trials included in this study was evaluated according to the PEDro scale, described in the Physiotherapy Evidence Database (www.pedro.org.au). The scale, composed of 11 items, was developed to classify the methodological quality of randomized clinical trials. Each item, except item 1, contributes one point to the full scale score, ranging from 0 to 10 points. The score used will be the one displayed at the website database. The scores of studies not included in the PEDro database or not punctuated will be performed by the authors of this study, based on the scale made available by the database.

\section{RESULTS}

The electronic search conducted by the authors resulted in 2485 articles. Of these, 2156 were excluded after reading the titles and 317 after reading the abstracts. Of the 12 potentially eligible papers remaining, one was excluded after reading the full text because it was not in fact a randomized clinical trial. Manual search did not return any jobs. Thus, a total of 11 articles were included in the present review ${ }^{(3,6,7,9-16)}$ (Figure 1).

The selected studies were listed in Table 1, all of them being randomized, with scores ranging from 5 to 8 on the PEDro scale. Samples ranged from 18 to 235 subjects, totaling 600 participants, including acute, subacute and chronic stroke. The participants had a mean age ranging from 18 to 69 years, with 376 men, all hemiparetic due to stroke.

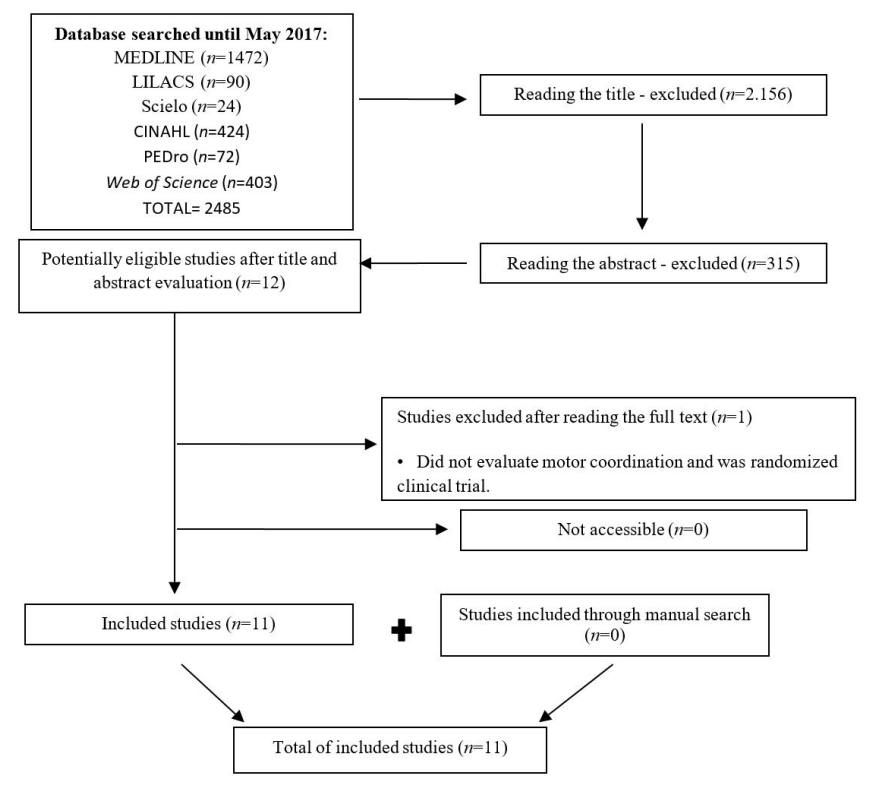

Figure 1. Flowchart for inclusion and exclusion of studies. 


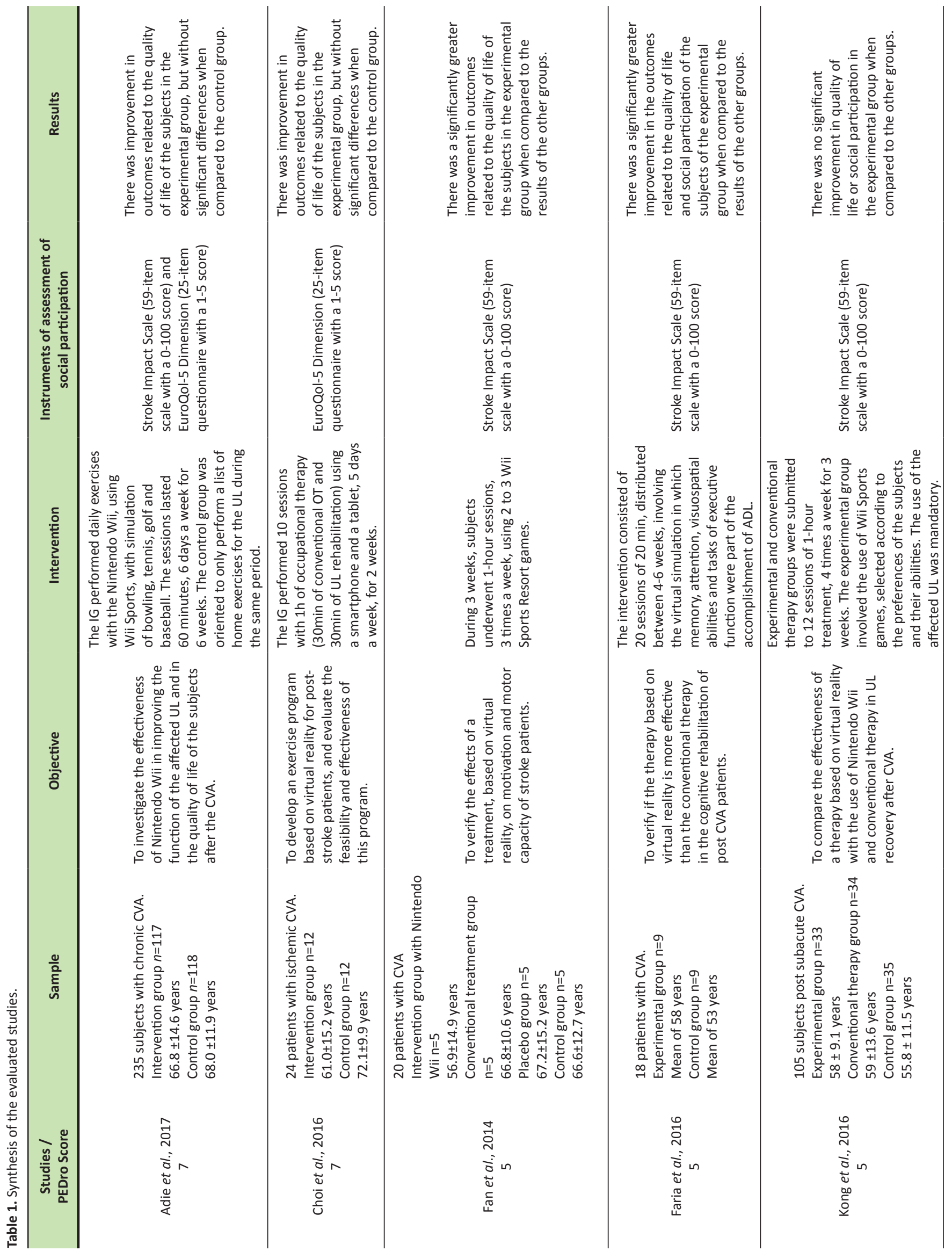




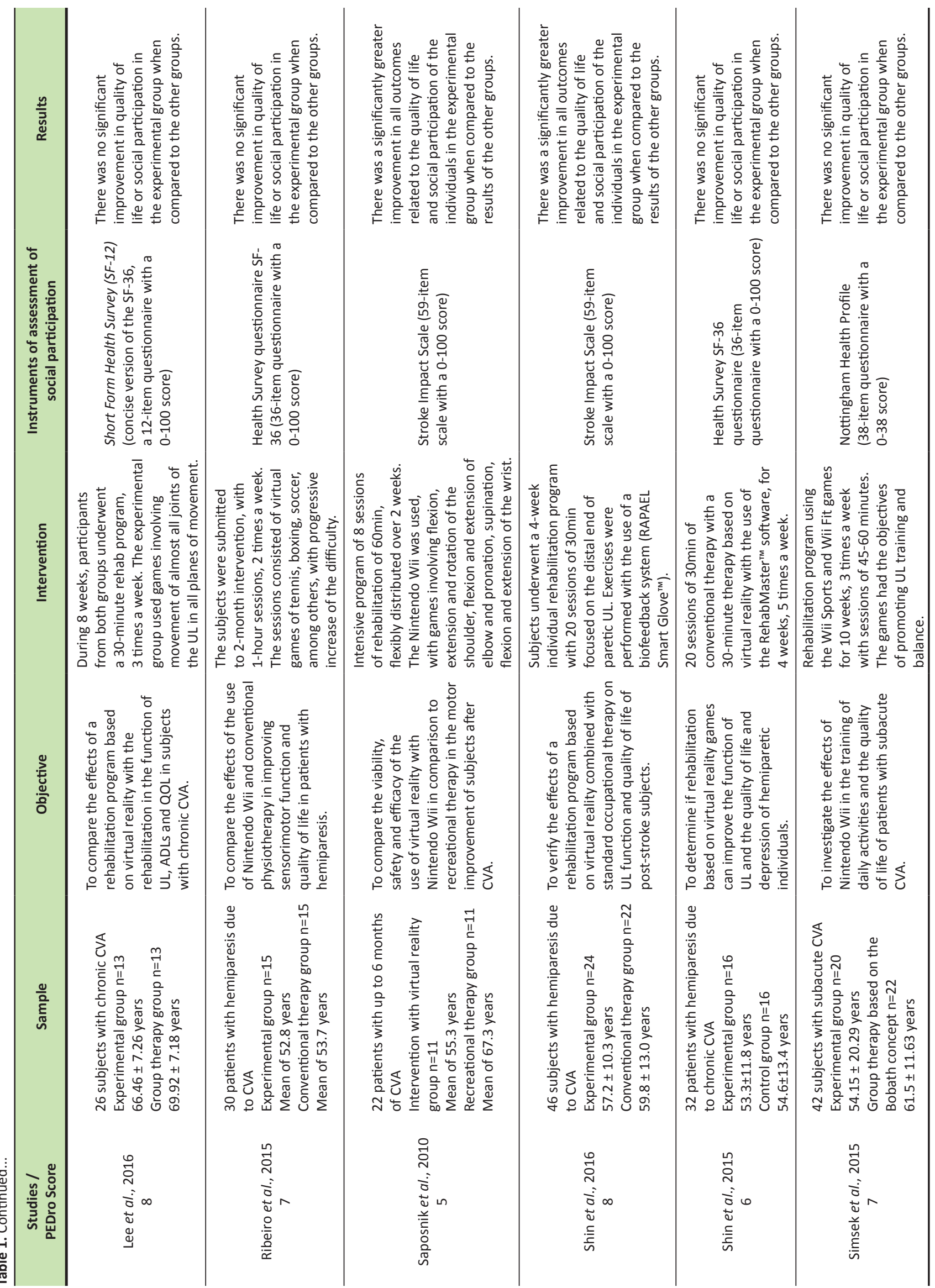


All subjects in the experimental groups were submitted to interventions that included some kind of virtual reality interface. In four of the eleven studies were used as a rehab feature the games of the Nintendo $\mathrm{Wii}^{\circledR}$. In the other studies, were used interfaces of non-commercial developed with the collaboration of the authors themselves. The minimum period of intervention reported was 2 weeks, and the maximum period was 8 weeks, with sessions of 30 and 60 minutes, 2 to 7 times a week. All participants performed tasks involving the compulsory use of upper limbs, as well as specific tasks of each study (gait, balance and others) according to the outcomes investigated by the authors. In addition to outcomes related to upper limb function, all studies also analyzed outcomes related to quality of life and social participation of the participants, which is the focus of interest of this review. The instruments used to assess social participation/quality of life were: Stroke Impact Scale (SIS), EuroQol-5 Dimension (EQ-5D 3L), Short Form Health Survey (SF-12), Health Survey SF-36 questionnaire (SF-36) and Nottingham Health Profile (NHP).

\section{DISCUSSION}

This study aimed to investigate the effects of the use of rehabilitation based on virtual reality in the recovery of the social participation of hemiparetic individuals after stroke. The analyzed studies show that although there seems to be benefits of using virtual reality in global aspects of hemiparetic patient recovery, such as in motor function, the results are still not consistent regarding social participation.

Of the included studies, four showed a significant improvement in the outcome of interest $\mathrm{t}^{(6,7,13,15)}$. In the others, either there was no improvement or it was not relevant when compared to the gains obtained by the subjects in the control groups or those submitted to other types of intervention that did not involve virtual reality training. Fan et al. attributed the gains of the patients to the motivational factor of virtual reality and to the fact that the games are closer to the actual movement required for the proposed tasks than other therapies $^{(6)}$. Whereas Faria et al. established a relationship between quality of life, social participation and adequate global cognitive functions, and also affirmed that positive results in the use of therapy based on virtual reality may be related to the fact that it represents a context closer to the real $^{(7)}$. In spite of this, the authors admited that the work lacks complementary information on the performance of patients in real factual tasks related to social participation after the intervention period. Lastly, Shin et al. cited the positive reinforcement of games through visual feedback and task specificity as relevant factors, but does not establish a direct relationship between these elements and the efficacy of virtual reality in improving quality of life ${ }^{(15)}$.

A possible explanation for the difference between the results found is the heterogeneity in the methods of the found articles. Although all clinical trials are randomized, there is considerable difference in sample size, virtual reality interface used and type of task or exercises performed in the interfaces. In addition, there are studies with only experimental and control groups and studies with at least one more group with individuals undergoing some other intervention. Among the studies that did not find significant differences between the analyzed groups, the reasons cited most frequently by the authors for this result are reduced sample sizes, short intervention time and no follow-up ${ }^{(10,12,14)}$, as well as lack of specificity of the proposed exercises ${ }^{(3)}$ and even stroke time ${ }^{(11,14)}$. According to Lee et al., the presence in the experimental group of subjects with a lesion time greater than seven years may have influenced the results, since, according to the authors' justification, gains in quality of life would tend to be higher in patients with a shorter stroke time ${ }^{(11)}$.

Other studies show similar results. In a review carried out by Palma et al. it was investigated the general effects of virtual reality use in post-stroke patients considering the domains of the International Classification of Functioning, Disability and Health ${ }^{(17)}$. It was included 54 studies with a total of 1811 participants. The authors verified that the results of the use of virtual reality are still not conclusive in what refers to the domains of social participation and activity. The work discusses that in addition to the recent use of this resource, the specific influence on social participation is complex because it involves cultural and motivational factors and it is not yet possible to establish a direct relationship between the improvement of the structural aspects of the body and the behavioral improvement, since there are still few studies that deal with the topic ${ }^{(17)}$.

In other populations and health conditions, such as in Parkinson's disease, Alzheimer's disease and cerebral palsy, the use of virtual reality specifically with the objective of promoting gains in quality of life and social participation also presents few studies available, and almost always the theme is treated as a secondary outcome. Most studies are still concerned with control of motor learning, muscle strength, gait quality and cortical reorganization, and results related to quality of life are still inconclusive ${ }^{(18,19)}$. They also cited the importance of virtual reality as a resource that may present differentials in relation to others during the rehabilitation process by promoting motivation, being more playful and possibly more functional, but they emphasize the need for further studies on the subject ${ }^{(18-20)}$.

The limitations of this study were the reduced sample of the studies, the moderate methodological quality, and very heterogeneous training protocols regarding equipment, time, frequency and duration of training, comparison, among others. Systematic and continuous comparison with a single resource or therapy or always with a placebo group would allow the observation of more consistent results regarding the real benefits of using virtual reality for the investigated outcomes. 


\section{CONCLUSION}

This systematic review demonstrates that the use of virtual reality can bring benefits to the rehabilitation of hemiparetic individuals, being a promising resource. However, in relation to social participation, which was the outcome of interest in this review, the results are still not conclusive, demonstrating the need for more studies on the subject, allowing reviews covering works with more homogeneous and adequate methodologies and, consequently, greater accuracy in results.

\section{AUTHORS' CONTRIBUTION}

KKPM elaborated the design of the study; LCQ, KKPM and PRA carried out the search and collection of data; LCQ, KKPM and PRA wrote and revised the manuscript. All authors read and approved the final manuscript.

\section{CONFLICT OF INTEREST}

The authors declare that there was no conflict of interest.

\section{REFERENCES}

1. MINISTÉRIO DA SAÚDE. Secretaria de Atenção à Saúde. Diretrizes de Atenção à Reabilitação da Pessoa com Acidente Vascular Cerebral. 2013

2. Kim N, Park Y, Lee B. Effects of community-based virtual reality treadmill training on balance ability in patients with chronic stroke. J Phys Ther Scie. 2015;27(3):655-8.

3. Adie K, Schofield C, Berrow M, Wingham J, Humfryes J, Pritchard C, et al. Does the use of Nintendo Wii Sports TM improve arm function? Trial of WiiTM in Stroke: A randomized controlled trial and economics analysis. Clin Rehabil. 2017;31(2):173-85.

4. Rodrigues-Baroni JM, Nascimento LR, Ada L, Teixeira-Salmela LF. Walking training associated with virtual reality-based training increases walking speed of individuals with chronic stroke: systematic review with metaanalysis. Braz J Phys Ther. 2014;18(6):502-12.

5. You SH, Jang SH, Kim YH, Hallett M, Ahn SH, Kwon YH. Virtual realityinduced cortical reorganization and associated locomotor recovery in chronic stroke. Stroke. 2005;36(6):1166-71.

6. Fan SC, Su FC, Chen SS, Hou WH, Sun JS, Chen KH, et al. Improved intrinsic motivation and muscle activation patterns in reaching task using virtual reality training for stroke rehabilitation: A pilot randomized control trial. J Med Biol Eng. 2014;34(4):399-407.

7. Faria AL, Andrade A, Soares L, I Badia SB. Benefits of virtual reality based cognitive rehabilitation through simulated activities of daily living: a randomized controlled trial with stroke patients. J Neuroeng Rehabil. 2016;13(1):96.
8. Impellizzeri FM, Bizzini M. Systematic review and meta-analysis: A primer. Int J Sport Phys Ther. 2012;7(5):493-503.

9. Choi YH, Ku J, Lim H, Kim YH, Paik NJ. Mobile game-based virtual reality rehabilitation program for upper limb dysfunction after ischemic stroke. Restor Neurol Neurosci. 2016;34(3):455-63.

10. Kong $\mathrm{KH}$, Loh YJ, Thia E, Chai A, Ng CY, Soh YM. Efficacy of a virtual reality commercial gaming device in upper limb recovery after stroke: $A$ randomized, controlled study. Top Stroke Rehabil. 2016;23(5):333-40.

11. Lee M, Son J, Kim J, Pyun SB, Eun SD, Yoon B. Comparison of individualized virtual reality-and group-based rehabilitation in older adults with chronic stroke in community settings: a pilot randomized controlled trial. Eur J Integ Med. 2016;8(5):738-46.

12. Ribeiro NMS, Ferraz DD, Pedreira É, Pinheiro Í, da Silva Pinto AC, Neto $\mathrm{MG}$, et al. Virtual rehabilitation via Nintendo $\mathrm{Wii}^{\circledR}$ and conventional physical therapy effectively treat post-stroke hemiparetic patients. Top Stroke Rehabil. 2015;22(4):299-305.

13. Saposnik G, Teasell R, Mamdani M, Hall J, Mcllroy W, Cheung D, et al. Effectiveness of virtual reality using Wii gaming technology in stroke rehabilitation. Stroke. 2010;41(7):1477-84.

14. Shin JH, Bog Park S, Ho Jang S. Effects of game-based virtual reality on health-related quality of life in chronic stroke patients: A randomized, controlled study. Comput Biol Med. 2015;63:92-98.

15. Shin JH, Kim MY, Lee JY, Jeon YJ, Kim S, Lee S. Effects of virtual realitybased rehabilitation on distal upper extremity function and health-related quality of life: a single-blinded, randomized controlled trial. J Neuroeng Rehabil. 2016;13(1):17.

16. Şimşek TT, Çekok K. The effects of Nintendo WiiTM-based balance and upper extremity training on activities of daily living and quality of life in patients with sub-acute stroke: a randomized controlled study. Int J Neurosci. 2016;126(12):1061-70.

17. Palma GC, Freitas TB, Bonuzzi GM, Soares MA, Leite PH, Mazzini NA, et al. Effects of virtual reality for stroke individuals based on the International Classification of Functioning and Health: a systematic review. Top Stroke Rehabil. 2017;24(4):269-78.

18. Lee JH, Ku J, Cho W, Hahn WY, Kim IY, Lee SM, et al. A virtual reality system for the assessment and rehabilitation of the activities of daily living. CyberPsychol Behav. 2003;6(4):383-8.

19. Mirelman A, Maidan I, Herman T, Deutsch JE, Giladi N, Hausdorff JM. Virtual reality for gait training: can it induce motor learning to enhance complex walking and reduce fall risk in patients with Parkinson's disease? J Gerontol A Biol Sci Med Sci. 2011;66(2):234-40.

20. Chiu HC, Kuo PW. Effects of virtual reality in children with cerebral palsy: A systematic review. Develop Med Child Neurol. 2015;40(3):136-44. 